\title{
Therapeutic HIV vaccines show promise
}

The world buzzed last week with news that an antiretroviral gel can halve the incidence of HIV infection in women (see doi:10.1038/ news.2010.363).

But a quieter buzz could be heard at the International AIDS Conference (AIDS 2010) in Vienna, where the gel results were unveiled. At a special session, included in the programme at the last minute, attendees heard the results of a handful of successful, but small, early-phase clinical trials for therapeutic vaccines - once thought to be a dead end for tackling HIV.

Normal vaccines are designed to prevent infections, but so far none has worked for HIV. Therapeutic vaccines, in contrast, aim to treat infected people - in the case of HIV, by boosting ravaged immune systems. Initial clinical trials in the 1990s were disappointing, however, and the vaccines fell from scientific fashion.

Drug combinations that decrease viral concentrations have become the main method of treating HIV, but they do not completely suppress the underlying disease. "They leave patients with a level of harmful immune activation, which can cause premature ageing," says Joep Lange, a clinical virologist from the University of Amsterdam and a former president of the International AIDS Society. “The therapeutic vaccine approach may help with this", he says, by modifying immune responses.

Lange is encouraged by the trial results, but cautions that the trials so far are small, and that even if the vaccines work they will never replace drugs. Some key AIDS researchers continue to believe that therapeutic vaccines will not prove helpful in the long run.

All of the vaccines, which were developed by several small biotechnology companies, modestly but significantly reduced viral levels in the blood of patients, who responded for months or longer. In some cases, the vaccines also increased levels of $\mathrm{CD} 4^{+} \mathrm{T}$ cells - the vital immune-regulator cells that HIV depletes. In theory, the vaccines would only need to be administered every few months.

Two of the phase 2 trials reported at the meeting focused on improving the efficiency of the immune system's dendritic cells. These are the cells that present foreign antigens - in this case, HIV proteins - to $T$ cells so that they can recognise and eliminate the invaders.

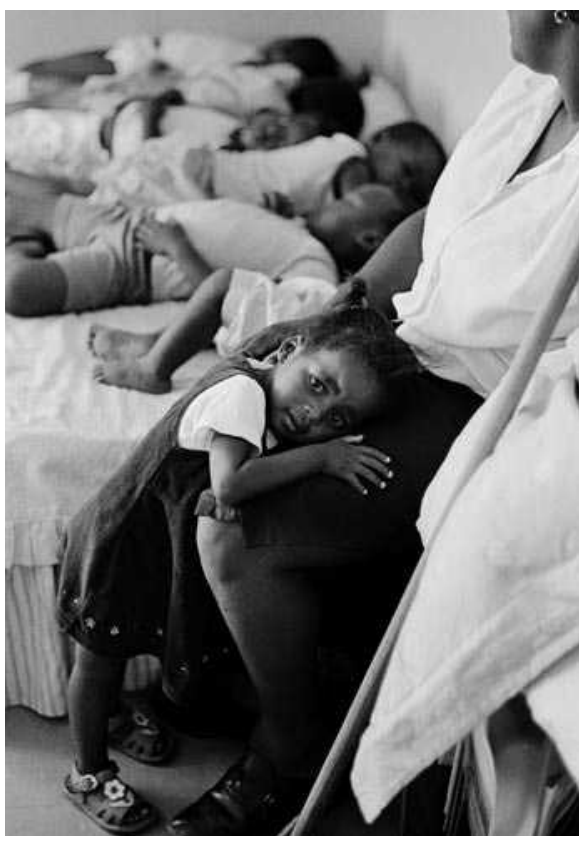

Could therapeutic vaccines help HIV patients in countries without ready access to drugs?

One approach, developed by Genetic Immunity, a biotechnology company based in Budapest, involves creating nanoparticles that contain selected pieces of RNA from the HIV virus. The preparation is applied to patients by means of a skin patch. The skin below the patch is first slightly damaged to attract precursors of dendritic cells, exposing them to 15 HIV proteins transcribed from the RNA.

The other tactic, from Argos Therapeutics of Durham, North Carolina, relies on tailor-made vaccines for each patient. The researchers extracted dendritic cells and viral RNA from patients, then loaded the cells with the RNA before putting them back into the same patient.

Therapeutic vaccines are normally tested in patients who are already undergoing drug treatment. To avoid the drugs confounding the results, patients are required to take a drug 'holiday' for the few months of the trial. But a placebo-controlled clinical trial by FIT Biotech of Tampere, Finland, broke with that model by recruiting 60 patients in South Africa who had never been treated with drugs.

The FIT vaccine comprises a combination of gene fragments designed to make the patient immune to six viral proteins. In around $80 \%$ of patients receiving treatment the virus was suppressed and $\mathrm{CD} 4^{+}$levels were maintained two years after therapy began.

The results are particularly relevant to countries such as South Africa, where many patients do not have easy access to drugs, says Eftyhia Vardas, a virologist at the University of the Witwatersrand, who ran the clinical trial in 2006 in Soweto, Johannesburg. Vardas recalls feeling like a 'maverick' when she agreed to take on the trial. At the time, the South African government officially denied that HIV caused AIDS, and her scientific colleagues didn't believe that therapeutic vaccines held any promise. She was sceptical herself, she says, having seen other trials fail.

"But you can't shut doors when options are so limited," she says. South Africa is home to 5.7 million HIV-infected people. A vaccine, says Vardas, "would help South Africa to be able to delay onset of AIDS and reduce infectivity by keeping viral loads low when drugs are not so widely available".

The ultimate value of the vaccines will only become clear as larger phase 3 trials roll out over the next few years. For now, leaders in AIDS research are cautious about the results. Anthony Fauci, director of the US National Institute of Allergy and Infectious Diseases (NIAID) in Bethesda, Maryland, says that he could imagine a role for "a good therapeutic vaccine" in patients who have been treated early in their disease, and therefore have only a small reservoir of HIV, and whose blood levels of HIV are completely suppressed by drugs. But he warns that because the vaccines caused only a modest decrease in viral load, using them in place of drugs could allow viruses to mutate beyond the control of the vaccine. "These early trials involve small numbers of people," adds Carl Dieffenbach, head of the NIAID AIDS division. "It would be wrong to foster false hopes."

But the Maryland-based advocacy group National Association of People with AIDS, whose vice-president of community affairs, Stephen Bailous, organized the special session, has championed the approach. "We need to have hopes and some of the therapeutic vaccines look really promising," says Bailous. "If we can't raise our hopes there, then where?"

Alison Abbott 